\title{
Biosimilars: a regulatory perspective from America
}

Jonathan Kay*

\begin{abstract}
Biosimilars are protein products that are sufficiently similar to a biopharmaceutical already approved by a regulatory agency. Several biotechnology companies and generic drug manufacturers in Asia and Europe are developing biosimilars of tumor necrosis factor inhibitors and rituximab. A biosimilar etanercept is already being marketed in Colombia and China. In the US, several natural source products and recombinant proteins have been approved as generic drugs under Section 505(b)(2) of the Food, Drug, and Cosmetic Act. However, because the complexity of large biopharmaceuticals makes it difficult to demonstrate that a biosimilar is structurally identical to an already approved biopharmaceutical, this Act does not apply to biosimilars of large biopharmaceuticals. Section 7002 of the Patient Protection and Affordable Care Act of 2010, which is referred to as the Biologics Price Competition and Innovation Act of 2009, amends Section 351 of the Public Health Service Act to create an abbreviated pathway that permits a biosimilar to be evaluated by comparing it with only a single reference biological product. This paper reviews the processes for approval of biosimilars in the US and the European Union and highlights recent changes in federal regulations governing the approval of biosimilars in the US.
\end{abstract}

Over the past decade, the availability of targeted biological therapies has revolutionized the treatment of rheumatoid arthritis, psoriatic arthritis, and ankylosing spondylitis and holds promise to expand treatment options for patients with systemic lupus erythematosus or other systemic autoimmune diseases. However, the significant cost of these medications creates a major barrier that limits universal access to these effective therapeutic agents. Whereas generic equivalents are commercially available for many small-molecule medications, such lower-cost

*Correspondence: jonathan.kay@umassmemorial.org

UMass Memorial Medical Center, Rheumatology Center, Memorial Campus, 119 Belmont Street, Worcester, MA 01605, USA alternatives to targeted biological therapies are not yet available in the US or the European Union.

Biopharmaceuticals are medications, predominantly proteins, that are manufactured using live organisms. These include blood and plasma products, non-recombinant proteins purified from their natural sources, recombinant proteins and monoclonal antibodies produced in cell culture, vaccines, and cultured cellular and tissue products [1]. 'Follow-on' protein products are those 'manufactured using biotechnology or derived from natural sources that are intended to be sufficiently similar to a' biopharmaceutical 'product or products' already approved by a regulatory agency [2]. These are called biosimilars and also have been referred to as biogenerics or biocomparables. A biosimilar product is defined in Section 351 of the Public Health Service (PHS) Act (42 USC $\mathbb{2 6 2}$ ) as one which is 'highly similar to the reference product notwithstanding minor differences in clinically inactive components' and for which there are 'no clinically meaningful differences between the biological product and the reference product in terms of safety, purity and potency of the product' [1]. The term 'biogeneric' is now obsolete because, unlike smallmolecule generic drugs, a biosimilar is never identical to its reference product.

A biosimilar etanercept, manufactured in China by $\mathrm{CP}$ Guojian Pharmaceutical Co., Ltd. (Shanghai), is already being marketed in China as Yisaipu [3] and in Colombia as Etanar [4]. Several biotechnology companies in Asia are also developing biosimilar versions of tumor necrosis factor inhibitors. Protalix Biotherapeutics, Inc. (Carmiel, Israel) is developing a biosimilar etanercept that is expressed in plant cells [5]. Mycenax Biotech (Taiwan) has completed early-phase clinical trials of a biosimilar etanercept in Southeast Asia: a phase I trial among 24 healthy subjects in South Korea and a phase I/II trial that enrolled 18 patients with rheumatoid arthritis in Taiwan [6]. Avesthagen (Bangalore, India) has received a patent from the Indian patent office for a biosimilar etanercept [7]. In South Korea, both Celltrion (Yeonsu-gu Incheon City) and Aprogen (Daejeon) are developing a biosimilar of infliximab [8] and LG Life Sciences (Seoul) is developing biosimilars of both etanercept and infliximab to treat rheumatoid arthritis and other inflammatory diseases [9]. 
Biosimilar versions of other targeted biological therapies for rheumatic diseases are also being developed. Since 2007, Dr. Reddy's Laboratories Ltd. (Hyderabad, India) has marketed Reditux, a biosimilar rituximab, for the treatment of non-Hodgkin's lymphoma and, more recently, as Reditux-RA for the treatment of rheumatoid arthritis [10]. Two other manufacturers of generic drugs are developing biosimilars of rituximab for the treatment of rheumatoid arthritis. Teva Pharmaceutical Industries Ltd. (Petach Tikva, Israel) is conducting a phase I/II trial comparing the pharmacokinetics, pharmacodynamics, safety, and preliminary efficacy of its biosimilar rituximab TL011 with those of MabThera ${ }^{\circ}$ in patients with active rheumatoid arthritis (ClinicalTrials.gov identifier NCT01123070) [11]. Sandoz, the generics division of Novartis (Basel, Switzerland), has also initiated a phase II clinical trial of its biosimilar rituximab GP2013, assessing similar parameters in patients with rheumatoid arthritis (ClinicalTrials.gov identifier NCT01274182) [12].Biosimilar versions of other targeted biological therapies for rheumatic diseases have also begun phase I pharmacokinetic and pharmacodynamic comparative clinical studies [13].

Although no biosimilars have yet been approved for treatment of a rheumatic disease, the prospect of their impending availability raises several concerns for the rheumatologist: (a) Will a biosimilar be as effective as the originally licensed biopharmaceutical? (b) Will a biosimilar be as safe as the originally licensed biopharmaceutical? (c) If a pharmacist substitutes a biosimilar for a prescribed biopharmaceutical, will the patient be adversely affected? (d) Will the availability of biosimilars reduce the high cost of targeted biological therapies for our patients?

Identical follow-on proteins of certain small peptides, for which the amino acid sequences have been determined, have been synthesized. However, most biosimilars are not identical to the original product but exhibit a range of structural similarities. Proteins produced by recombinant DNA technology are highly similar to the original proteins and share the primary amino acid sequence but differ with respect to glycosylation and other post-translational modifications. Other nonrecombinant proteins that are purified from their natural sources are generally similar to the original biopharmaceuticals but, in addition to exhibiting different posttranslational modifications, may have slightly different amino acid sequences.

Distinct from biosimilars are 'second-generation' biopharmaceuticals that are structurally different from the originally licensed biopharmaceutical and that are intended to improve performance while preserving the mechanism of action. However, these second-generation biopharmaceuticals are not usually considered to be follow-on protein products. Major changes in the manufacture of approved protein biopharmaceuticals also introduce a degree of uncertainty similar to that raised by biosimilars. However, in these situations, the original manufacturer maintains access to detailed information that pertains to the changes in production and that would not be available to the manufacturer of a biosimilar.

Manufacturing changes are frequently made by the original developers of protein biopharmaceuticals and their partners. Some production changes have resulted in decreased immunogenicity. When interferon $\beta 1$ a was produced by Biogen Idec (Weston, MA, USA) in a new cell line, the resulting product, Avonex, had decreased immunogenicity compared with the interferon $\beta 1 \mathrm{a}$ that had been produced in the original Chinese hamster ovary cell line by Bioferon Biochemische Substanzen $\mathrm{GmbH}$ \& Co. (Laupheim, Germany) [2,14]. On the other hand, other changes in formulation or packaging have resulted in increased immunogenicity with clinical consequences. A change in the formulation of Eprex (recombinant human erythropoietin manufactured by Ortho Biotech, part of Janssen, Toronto, ON, Canada), switching the protein stabilizer from human serum albumin to the detergent polysorbate 80 and introducing a new packaging system for subcutaneous administration using a prefilled syringe with a rubber plunger, resulted in the formation of anti-erythropoietin antibodies that crossreacted with endogenous erythropoietin $[15,16]$. These cross-reactive antibodies caused 175 patients receiving this new formulation of Eprex to develop pure red cell aplasia between 1998 and 2004 [17]. Thus, a change in the manufacture of a biopharmaceutical may result in an outcome that is either beneficial or detrimental.

In the US, the process whereby protein and peptide drugs, such as enzymes and hormones, are approved and licensed by the US Food and Drug Administration (FDA) is governed by Section 505 of the Food, Drug, and Cosmetic (FD\&C) Act (21 US Code [USC] \$ 355) [18]. In contrast, the process by which large, complex biopharmaceuticals, including the targeted biological agents used to treat rheumatic diseases, are approved and licensed is specified in Section 351 of the PHS Act (42 USC $\$$ 262) [1]. Each of these federal statutes mandates that the pharmaceutical manufacturer consult with the FDA, which acts as the representative of the secretary of the US Department of Health and Human Services (HHS), prior to commencing the clinical development program to decide upon the 'design and size of clinical trials of a new drug' that will be necessary for the drug to gain approval [18].

The present system of generic drugs in the US was established by the Drug Price Competition and Patent Term Restoration Act of 1984 (also known as the HatchWaxman Act) (Public Law 98-417). This Act amended the FD\&C Act and provided two abbreviated pathways 
for the approval of generic drugs, including natural source products and recombinant proteins [18]. Section 505(b)(2) eliminates the unnecessary duplication of preclinical and some human studies in the New Drug Application (NDA) for a generic drug by allowing direct comparison of the generic drug with a product already approved for the same indication in brief clinical trials of 3 to 6 months in duration. Section 505(j) sets forth the process by which the producer of a generic drug that is identical to a previously approved product can file an Abbreviated New Drug Application (ANDA) to seek FDA approval of the generic compound. An ANDA allows the applicant to rely on the FDA's previous finding of safety and efficacy for the already approved drug.

As specified in Section 505(b)(2) of the FD\&C Act, the current FDA process for evaluating biosimilar protein and peptide drugs in a marketing application allows the follow-on applicant to rely, in part, on available knowledge about the safety and effectiveness of the structurally similar, already approved product. Public access to information about the manufacture of the original product, such as the source material used and any chemical modifications that may have been introduced, facilitates this assessment. However, this evaluation still requires both preclinical and clinical studies to determine the safety, purity, and potency of the biosimilar. In this way, several natural source products and recombinant proteins - for example, natural bovine testicular and recombinant human hyaluronidase, recombinant salmon calcitonin, recombinant human glucagon, and recombinant human somatropin - have been approved as generic drugs under Section 505(b)(2) of the FD\&C Act. The approval of each relied upon structural characterization, comparative pharmacokinetic and pharmacodynamic data, and immunogenicity and safety data [2].

Because of the complexity of large biopharmaceuticals, the manufacturer of a follow-on protein product has difficulty in demonstrating that it is identical to an already approved product. Thus, the abbreviated approval pathway specified in Section 505(j) of the FD\&C Act is not usually available for protein products.

An abbreviated pathway for the approval of biosimilars in the European Union has been proposed by the Committee for Medicinal Products for Human Use of the European Medicines Agency (EMA). In 2005, guidance was issued outlining the data necessary 'to substantiate the claim of similarity used as the basis for a marketing authorization application (MAA)' for large biopharmaceuticals [19]. Shortly thereafter, the EMA published a guideline that proposed the assessment expected before consideration of an MAA for 'similar biological medicinal products containing biotechnology-derived proteins as active substance' [20]. Both preclinical in vitro assays and in vivo animal studies and clinical studies in patients are required to compare the biosimilar with the reference product. If available, single- and multiple-dose pharmacokinetic studies and pharmacodynamic studies using biomarkers relevant to the clinical efficacy of the drug are necessary 'to demonstrate clinical comparability' between the biosimilar and the reference product. In most cases, 'comparative clinical trials' are also needed to demonstrate clinical equivalence between the biosimilar and the already approved reference product and to assess potential immunogenicity with chronic dosing. Because subtle differences between the biosimilar and the reference product may result in an unanticipated loss of efficacy or adverse reactions, careful post-approval pharmacovigilance monitoring is expected. The Biosimilar Medicinal Products Working Party of the EMA is in the process of revising these guidelines and preparing other guidelines regarding the assessment of individual biosimilar protein drugs [21].

In the US, an abbreviated approval pathway for large biopharmaceuticals was established by the recent ratification of the Patient Protection and Affordable Care (PPAC) Act of 2010 (H.R. 3590). This bill was passed by the US Senate on 24 December 2009 and by the US House of Representatives by a very narrow margin (219 for and 212 against) on 21 March 2010. The following day, President Barack Obama signed this Act into law as Public Law 111-148. Section 7002 of the Patient Protection and Affordable Care Act, which is referred to as the Biologics Price Competition and Innovation Act of 2009, amends Section 351 of the PHS Act to create an abbreviated Biologic License Application (BLA) for 'highly similar' biological products; the abbreviated BLA permits a follow-on biological product to be evaluated against only a single reference biological product [22]. To be considered for an abbreviated BLA, the biosimilar must have the same presumed mechanism of action, route of administration, dosage form, and potency as the innovator product. It may only be reviewed and approved for indications for which the FDA already has approved the innovator product.

Data obtained from analytical and animal studies and from at least one clinical trial, which must be conducted in patients with a disease for which the innovator product is licensed, are to be used to demonstrate that a follow-on biological product is 'highly similar' to the reference product, 'notwithstanding minor differences in clinically inactive components'. The clinical study must demonstrate 'safety, purity, and potency' of the follow-on biological product, evaluate its pharmacokinetics and pharmacodynamics, and include an assessment to establish that it is not more immunogenic than the innovator reference product [22]. Once these studies have been completed, the HHS secretary must license an abbreviated BLA if she determines that the information 
submitted is sufficient to show that the follow-on biological is 'biosimilar' to or 'interchangeable' with the innovator product [22]. Prior to licensing, the applicant must also consent to inspection of the manufacturing facility to ensure that it follows 'good manufacturing practice'. However, at her 'discretion', the HHS secretary may 'waive any of these requirements on a case-by-case basis'. Regardless, the same risk evaluation and mitigation strategies (REMSs) required of the innovator biologic agent licensed under the PHS Act are applied to the biosimilar [22].

The FDA has established the Biosimilar Implementation Committee - co-chaired by Janet Woodcock, director of the Center for Drug Research and Evaluation, and Karen Midthun, acting director of the Center for Biologics Evaluation and Research - to determine the process by which the FDA will approach the evaluation, review, and approval of abbreviated BLAs, as mandated by the Biologics Price Competition and Innovation Act [23]. It remains to be specified whether the clinical trials required for an abbreviated BLA may demonstrate noninferiority of the biosimilar to the reference product or must prove superiority of the biosimilar to placebo or to another active comparator. Acceptable measures of efficacy and the suitability of biomarkers as surrogates for clinical endpoints also remain to be clarified.

A follow-on biological product is defined as being 'interchangeable' with the innovator product if it is 'biosimilar' to that product and can be 'expected to produce the same clinical result' as that product in 'any given patient' and if there is no greater risk of safety or diminished efficacy when alternating or switching between the follow-on biological and innovator products than when using the innovator product with no switch. If these conditions for interchangeability are met, the HHS secretary is required to determine that the biosimilar is 'interchangeable' with the reference product at the time of approval [22]. If so, that biosimilar may be substituted for the prescribed innovator product by someone other than the health-care provider who issued the original prescription. In such a situation, a pharmacist would be allowed to substitute the 'interchangeable' biosimilar for a prescribed targeted biological therapy without involving the patient's rheumatologist. However, unlike small-molecule drugs, a biopharmaceutical that is repeatedly interchanged with a similar biological agent may exhibit immunogenicity that could compromise the efficacy and safety of both medications. The clinical consequences of neutralizing antibodies developing to a non-redundant protein with an important biological function, such as erythropoietin, may be severe [15]. Thus, it is extremely important that there not be frequent switching between the original protein product and the biosimilar, because even subtle differences, such as impurities introduced during manufacturing, may trigger an immune response to the biosimilar.

The Biologics Price Competition and Innovation Act provides the manufacturer of the innovator product with economic protection by granting a period of 'exclusivity' during which follow-on products may not be approved [22]. A BLA for approval of a follow-on biological product may not be submitted for 4 years after the reference product was initially approved. The FDA may not approve a BLA for a follow-on biological product until 12 years after the reference product was first licensed. No additional period of exclusivity will be granted to a previously licensed biologic product when subsequent applications are made for a new indication, route of administration, dosage form, or dosing strength. However, each of the periods of exclusivity may be extended by 6 months if studies of the innovator biological product in the pediatric population are requested by the HHS secretary and carried out.

To encourage the development of biosimilars, the Biologics Price Competition and Innovation Act grants 1 year of exclusive marketing rights to the first follow-on biological that is approved as being 'interchangeable' with a reference product [22]. If patent litigation between the manufacturers of the follow-on and innovator products is ongoing, this period of exclusivity may be extended for up to 42 months.

The recently enacted Biologics Price Competition and Innovation Act establishes an abbreviated pathway whereby biosimilars can be evaluated and approved in an accelerated manner. However, the details of the process by which the FDA will implement this pathway have yet to be worked out. As several biosimilar versions of targeted biological therapies for treatment of rheumatoid arthritis are being developed in Asia and Europe, the abbreviated BLA established by this Act sets up a regulatory mechanism for their approval in the US.

The Biologics Price Competition and Innovation Act mandates that, to be approved, biosimilars be as safe and effective as the originally licensed biopharmaceutical. Although it would be ideal to reduce the cost of development of biosimilars by abbreviating the BLA pathway and to pass that cost savings on to the patient, it is yet to be shown that fewer clinical trials than were required of the innovator biopharmaceutical will provide adequate data regarding the safety and efficacy of a biosimilar. Meeting conditions of 'interchangeability' will ensure that the patient should not be adversely affected if a pharmacist substitutes a biosimilar for a prescribed biopharmaceutical. However, it is not readily apparent that many biosimilars will be determined to be 'interchangeable' with the reference product. Only time and experience will validate the adequacy of this new regulatory pathway for approval of biosimilars in the US. 


\section{Abbreviations}

ANDA, Abbreviated New Drug Application; BLA, Biologic License Application EMA, European Medicines Agency; FD\&C, Food, Drug, and Cosmetic; FDA, US Food and Drug Administration; HHS, US Department of Health and Human Services; MAA, marketing authorization application; NDA, New Drug Application; PHS, Public Health Service; USC, US Code.

\section{Competing interests}

The author declares that he has no competing interests.

Published: 12 May 2011

\section{References}

1. Public Health Service Act Sec. 262. Regulation of biological products [http://www.fda.gov/Regulatorylnformation/Legislation/ucm149278.htm].

2. Woodcock J, Griffin J, Behrman R, Cherney B, Crescenzi T, Fraser B, Hixon D, Joneckis C, Kozlowski S, Rosenberg A, Schrager L, Shacter E, Temple R, Webber $\mathrm{K}$, Winkle H: The FDA's assessment of follow-on protein products: a historical perspective. Nat Rev Drug Discov 2007, 6:437-442.

3. Yisaipu [http://www.cpgj-pharm.com/en/product_patient. asp?proid=22\&action=intro].

4. Rondon F, Bautista A, Salazar JC, Casas N, Santos P, Vargas F, Marquez J: Etanar therapy in real-life patients with rheumatoid arthritis [abstract]. Arthritis Rheum 2010, 62 (Suppl 10):1811.

5. Pipeline products [http://www.protalix.com/pipeline_products.html]

6. Biosimilar TuNEX ${ }^{\circledR}$ completes Phase I/II clinical trial in Taiwan, Phase I in Korea [http://www.mycenax.com.tw/webe/html/02news/news_show. aspx?page=1].

7. Singh K: Avesthagen gets patent for Enbrel biosimilar. Economic Times. 3 September 2010:6.

8. Korea's Celltrion and Aprogen in race to sell biosimilars in Japan [http://sis.windhover.com/buy/abstract.php?id=28101102003]

9. LG Life Sciences' Pipeline Overview [http://thinklgls.com/rnd/pipeline].

10. Dr. Reddy's Marketed Pharmaceutical Products [http://www.drreddys.com/ products/bio_mproducts.html\#].

11. TL011 in severe, active rheumatoid arthritis patients [http://Clinicaltrials. gov/ct2/show/NCT01123070].

12. GP2013 in the treatment of RA patients refractory to or intolerant of standard therapy [http://www.clinicaltrials.gov/ct2/show/NCT01274182].

13. View Opportunities [http://www.sourcegenerics.com/viewAllListing.asp].

14. Rudick RA, Simonian NA, Alam JA, Campion M, Scaramucci JO, Jones W, Coats ME, Goodkin DE, Weinstock-Guttman B, Herndon RM, Mass MK, Richert JR,
Salazar AM, Munschauer FE 3rd, Cookfair DL, Simon JH, Jacobs LD: Incidence and significance of neutralizing antibodies to interferon beta-1a in multiple sclerosis. Multiple Sclerosis Collaborative Research Group (MSCRG). Neurology 1998, 50:1266-1272.

15. Casadevall N, Nataf J, Viron B, Kolta A, Kiladjian JJ, Martin-Dupont P, Michaud P, Papo T, Ugo V, Teyssandier I, Varet B, Mayeux P: Pure red-cell aplasia and antierythropoietin antibodies in patients treated with recombinant erythropoietin. N Engl J Med 2002, 346:469-475

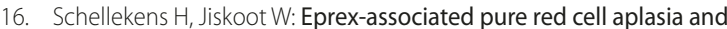
leachates. Nat Biotechnol 2006, 24:613-614.

17. Bennett $C L$, Luminari S, Nissenson AR, Tallman MS, Klinge SA, McWilliams N, McKoy JM, Kim B, Lyons EA, Trifilio SM, Raisch DW, Evens AM, Kuzel TM, Schumock GT, Belknap SM, Locatelli F, Rossert J, Casadevall N: Pure red-cell aplasia and epoetin therapy. N Engl J Med 2004, 351:1403-1408.

18. Federal Food, Drug, and Cosmetic Act (FD\&C Act) SEC. 505. [21 USC §355] New Drugs [http://www.fda.gov/Regulatorylnformation/Legislation/ FederalFoodDrugandCosmeticActFDCAct/ FDCActChapterVDrugsandDevices/ucm 108125.htm].

19. Committee for Medicinal Products for Human Use: Guideline on similar biological medicinal products. London: European Medicines Agency; 2005.

20. Committee for Medicinal Products for Human Use: Guideline on similar biological medicinal products containing biotechnology-derived proteins as active substance: non-clinical and clinical issues. London: European Medicines Agency; 2006.

21. European Medicines Agency: Work plan for the biosimilar medicinal products working party (BMWP) 2011. London: European Medicines Agency; 2010

22. H. R. 3590 - 686. Patient Protection and Affordable Care Act. Title VIIImproving Access to Innovative Medical Therapies. Subtitle A-Biologics Price Competition and Innovation. Sec. 7002. Approval Pathway for Biosimilar Biological Products [www.fda.gov/downloads/Drugs/ GuidanceComplianceRegulatoryInformation/UCM216146.pdf ].

23. Implementation of the Biologics Price Competition and Innovation Act of 2009 [http://www.fda.gov/Drugs/GuidanceComplianceRegulatorylnformation/ ucm215089.htm].

doi:10.1186/ar3310

Cite this article as: Kay J: Biosimilars: a regulatory perspective from America. Arthritis Research \& Therapy 2011, 13:112. 